\title{
FIXED POINT RESULTS IN COMPLEX VALUED RECTANGULAR EXTENDED $b$-METRIC SPACES WITH APPLICATIONS
}

\author{
NAIMAT ULLAH AND MOHAMMED SHEHU SHAGARI*
}

\begin{abstract}
In this article, two new fixed point results in the framework of complex-valued rectangular extended $b$-metric space are established. Our results include as special cases, some well-known results in the corresponding literature. We provide nontrivial examples and an existence theorem of a Fredholm type integral equation to support our assertions and to indicate a usability of the results presented herein.
\end{abstract}

MSC(2010): 46S40, 47H10, 54H25.

Keywords: Complex valued metric; Complex Valued Rectangular Extended $b$-metric; Fixed point; Integral equation.

\section{Introduction and Background}

The Banach contraction principle [9] is the first most well-known, simple and versatile classical result in fixed point theory with metric space structure. More than a handful of literature embrace applications and generalizations of this principle in different directions, for example, by weakening the hypotheses, employing different mappings and various forms of metric spaces. In this context, the work of Taskovic [21] is handy for recollecting various modifications of Banach type contractive definitions.

The study of new spaces and their properties have been an interesting topic among the mathematical research community. In this direction, the notion of $b$-metric spaces is presently flourishing. The idea commenced with the work of Bakhtin [8] and Bourbaki [10]. Later on, Czerwik [11] gave a postulate which is weaker than the classical triangle inequality and formally established a $b$-metric space with a view of improving the Banach fixed point theorem. Meanwhile, the notion of $b$-metric spaces has gained enormous generalizations, see, for example, $[15,19,20]$. For a recent short survey on basic concepts and results in fixed point theory in the framework of $b$-metric spaces, we refer the interested reader to Karapinar [17]. Along the line, Branciari [24] invented the concept of rectangular metric space by changing

Date: Received: November 10, 2020 , Accepted: December 27, 2020.

${ }^{*}$ Corresponding author. 
the triangular inequality. Recently, George et al. [22] initiated the notion of rectangular $b$-metric space as a generalization of metric and $b$-metric spaces, and consequently they proved analogues of Banach and Kannan fixed point theorems. Meanwhile, many researchers are working under these scenarios and deducing important results.

It is a fact that fixed point theorems concerning rational contractions cannot be extended or even meaningless in cone metric spaces. To remedy this obstacle, Azam et al. [4] introduced the concept of complex valued metric spaces and established sufficient conditions for the existence of common fixed points of a pair of mappings satisfying contractive type conditions involving rational inequalities. However, it is noteworthy that complex valued metric space is a special family of cone metric spaces. But, the definition of a cone metric is based on the underlying Banach space which is not a division ring. Therefore, many results of both linear and non-linear analysis concerning divisions cannot be discussed in cone metric spaces. On this development, the study of fixed point theorems concerning rational inequalities in complex valued metric spaces have been growing geometrically (see, for example, [1, 2, 5, 6, 12, 13, 18, 23]). In 1989, Rao [20] introduced the concept of fixed point results of complex valued $b$-metric spaces, which is broader than complex valued metric spaces. However, every complex valued $b$-metric space is a cone $b$-metric space over Banach algebra $\mathbb{C}$ in which the cone is normal with the coefficient of normality $K=1$, and where the cone has non-empty interior (that is, solid cone). Following [20], various authors have demonstrated fixed point results for different mappings fulfilling rational inequalities with regards to complex valued $b$-metric spaces (see, for instance, $[3,7])$.

Following the above developments, in this article, two new fixed point theorems in the setting of complex-valued rectangular extended $b$-metric space are discussed. Our results include as special cases, the main results of Banach [9], Czerwick [11], Kannan [16], George et al. [22], and a few important others in the comparable literature. We provide nontrivial examples and an existence theorem of Fredholm type integral equation to support our assertions and to indicate a usability of the results presented herein.

\section{Preliminaries}

In this section, we recall some specific concepts which are necessary for the presentation of our main results.

Definition 2.1. [4] Let $\mathbb{C}$ be the set of all complex numbers and $z_{1}, z_{2} \in \mathbb{C}$. The partial order on $\mathbb{C}$ is defined as:

$z_{1} \preceq z_{2}$, if and only if $\operatorname{Re}\left(z_{1}\right) \leq \operatorname{Re}\left(z_{2}\right)$ and $\operatorname{Im}\left(z_{1}\right) \leq \operatorname{Im}\left(z_{2}\right)$. This implies that $z_{1} \preceq z_{2}$ if one of the below conditions is fulfilled:

(i) $\operatorname{Re}\left(z_{1}\right)=\operatorname{Re}\left(z_{2}\right), \operatorname{Im}\left(z_{1}\right)<\operatorname{Im}\left(z_{2}\right)$,

(ii) $\operatorname{Re}\left(z_{1}\right)<\operatorname{Re}\left(z_{2}\right), \operatorname{Im}\left(z_{1}\right)=\operatorname{Im}\left(x_{2}\right)$, 
(iii) $\operatorname{Re}\left(z_{1}\right)<\operatorname{Re}\left(z_{2}\right), \operatorname{Im}\left(z_{1}\right)<\operatorname{Im}\left(z_{2}\right)$,

(iv) $\operatorname{Re}\left(z_{1}\right)=\operatorname{Re}\left(z_{2}\right), \operatorname{Im}\left(z_{1}\right)=\operatorname{Im}\left(z_{2}\right)$.

Definition 2.2. [4] Let $X$ be a non-empty set. If the mapping $\psi: X \times X \rightarrow$ $\mathbb{C}$ satisfies the following conditions :

(i) $0 \preceq \psi(x, y)$ and $\psi(x, y)=0 \Longleftrightarrow x=y$;

(ii) $\psi(x, y)=\psi(y, x)$;

(iii) $\psi(x, y) \preceq \phi(x, z)+\psi(z, y), \forall x, z, y \in X$,

then $\psi$ is known as a complex valued metric on $X$, and the pair $(X, \psi)$ is said to be a complex valued metric space.

Example 2.3. Let $X=X_{1} \cup X_{2}$, where

$$
X_{1}=\{z \in \mathbb{C}: \operatorname{Re}(z) \geq 0 \text { and } \operatorname{Im}(z)=0\}
$$

and

$$
X_{2}=\{z \in \mathbb{C}: \operatorname{Re}(z)=0 \text { and } \operatorname{Im}(z) \geq 0\} .
$$

Define $\psi: X \times X \longrightarrow \mathbb{C}$ as follows:

$$
\psi\left(z_{1}, z_{2}\right)= \begin{cases}\frac{2}{3}\left|x_{1}-x_{2}\right|+\frac{i}{2}\left|x_{1}-x_{2}\right|, & \text { if } z_{1}, z_{2} \in X_{1} \\ \frac{1}{2}\left|y_{1}-y_{2}\right|+\frac{i}{2}\left|y_{1}-y_{2}\right|, & \text { if } z_{1}, z_{2} \in X_{2} \\ \left(\frac{1}{2} y_{1}+\frac{2}{3} x_{2}\right)+i\left(\frac{1}{3} y_{1}+\frac{1}{2} x_{2}\right), & \text { if } z_{1} \in X_{1}, z_{2} \in X_{2}\end{cases}
$$

where $z_{1}=x_{1}+i y_{1}$ and $z_{2}=x_{2}+i y_{2}$. Then, $(X, \psi)$ is a complex valued metric space.

Definition 2.4. [14] Let $X$ be a non-empty set and $\theta: X \times X \rightarrow[1, \infty)$ be a function. Define a function $\psi: X \times X \rightarrow \mathbb{C}$ such that for all $x, y, z \in X$, the following conditions hold:

(i) $0 \preceq \psi(x, y)$ and $\psi(x, y)=0$ if and only if $x=y$;

(ii) $\psi(x, y)=\phi(y, x)$;

(iii) $\psi(x, y) \preceq \tau[\psi(x, z)+\psi(z, y)]$,

where $\tau \geq 1$. Then $\psi$ is called a complex valued $b$-metric on $X$ and the pair $(X, \psi)$ is called a complex valued $b$-metric space.

Example 2.5. [20] Let $X=[0,1]$. Define a mapping $\psi: X \times X \rightarrow \mathbb{C}$ by

$$
\psi(x, y)=|x-y|^{2}+i|x-y|^{2}
$$

for all $x, y \in X$. Then $(X, \psi)$ is a complex valued $b$-metric space with $\tau=2$.

Definition 2.6. [22] Let $X$ be a non-empty set and the mapping $\psi: X \times$ $X \rightarrow[0, \infty)$ satisfies:

(i) $\psi(x, y)=0, \Leftrightarrow x=y$ for all $x, y \in X$,

(ii) $\psi(x, y)=\psi(y, x)$ for all $x, y \in X$,

(iii) $\psi(x, y) \leq \tau[\psi(x, u)+\psi(u, v)+\psi(v, y)]$ for all $x, y \in X$, and $u, v \in$ $X-\{x, y\}$ with $\tau \geq 1$. Then $\psi$ is called a rectangular $b$-metric on $X$ and the pair $(X, \psi)$ is known as rectangular $b$-metric spaces.

We now introduce the concept of complex valued rectangular extended $b$-metric space as follows. 
Definition 2.7. Let $X$ be a non-empty set and $\theta: X \times X \rightarrow[1, \infty)$ be a function. Then, the function $\psi: X \times X \rightarrow \mathbb{C}$ is known as a complex valued rectangular extended $b$-metric space if the following conditions are satisfied for all $x, y, z \in X$ :

(i) $0 \preceq \psi(x, y)$ and $\psi(x, y)=0$ if and only if $x=y$;

(ii) $\psi(x, y)=\psi(y, x)$;

(iii) $\psi(x, z) \preceq \theta(x, z)[\psi(x, u)+\psi(u, v)+\psi(v, z)]$; for all $x, y \in X$ and all distinct points $u, v \in X-\{x, z\}$.

Then, the pair $(X, \psi)$ is known as a complex valued rectangular extended $b$-matric space.

Note that every complex-valued metric space is a complex-valued rectangular metric space, every complex-valued rectangular metric space is a complex-valued rectangular $b$-metric space and every complex-valued rectangular $b$-metric space is a complex-valued rectangular extended $b$-metric space. However the converse of the above implications is not always true. The next example illustrates this observation.

Example 2.8. Let $X=[0, \infty), \theta(x, y)=1+x+y$ for all $x, y \in X$ and define $\psi: X \times X \longrightarrow \mathbb{C}$ as follows:

$$
\psi(x, y)= \begin{cases}0, & \text { if } x=y \\ \frac{i \tau}{3}, & \text { if } x, y \in[0,10) \\ \frac{i}{2(4+\tau)}, & \text { if } x \text { or } y \notin[0,10),\end{cases}
$$

where $\tau \geq 1$. Then $(X, \psi)$ is a complex-valued rectangular extended $b$-metric space. However, $\psi$ is not a complex-valued rectangular $b$-metric on $X$. To see this, let $x=1, z=5$ and $u=v=10$. Then, for all $\tau \geq 1$,

$$
\begin{aligned}
\psi(x, z)=\frac{\tau i}{3} & >\tau\left(\frac{i}{4+\tau}\right) \\
& =\tau[\psi(x, u)+\psi(u, v)+\psi(v, z)] .
\end{aligned}
$$

The following two lemmas is adapted after similar results in [4].

Lemma 2.9. Let $(X, \psi)$ be a complex valued rectangular extended b-metric space and $\left\{x_{p}\right\}_{p \geq 1}$ be a sequence in $X$. Then, $\left\{x_{p}\right\}_{p \geq 1}$ is a convergent sequence if and only if $\left|\psi\left(x_{p}, x\right)\right| \rightarrow 0$ as $p \rightarrow \infty$.

Lemma 2.10. Let $(X, \psi)$ be a complex valued rectangular extended b-metric space and $\left\{x_{p}\right\}_{p \geq 1}$ be a sequence in $X$. Then $\left\{x_{p}\right\}_{p \geq 1}$ is a Cauchy sequence if and only if $\left|\psi\left(x_{p}, x_{q}\right)\right| \rightarrow 0$ as $p, q \rightarrow \infty$.

Our main result is a combination of the next two results.

Theorem 2.11. [9] Let $(X, \psi)$ be a complete metric space and $T: X \rightarrow X$ be a contraction mapping. Then $T$ has a unique fixed point, that is there is one and only one point $x \in X$ such that $T(x)=x$. 
Theorem 2.12. [22] Let $(X, \psi)$ be complete rectangular b-metric space with the coefficient $\tau \geq 1$ and $T: X \rightarrow X$ be a mapping satisfying: $\psi(T x, T y) \preceq$ $\lambda \psi(x, y)$, for all $x, y \in X$; where $\lambda \in\left[0, \frac{1}{\tau}\right]$. Then $T$ has a unique fixed point.

\section{Main Results}

Our first main result, unifying and improving Theorems 2.11 and 2.12 runs as follows.

Theorem 3.1. Let $(X, \psi)$ be a complete complex valued extended rectangular $b$-metric space, $\theta: X \times X \rightarrow[1, \infty)$ be a function and let $T: X \rightarrow X$ be a mapping satisfying:

$$
\psi(T x, T y) \preceq \lambda \psi(x, y)
$$

for all $x, y \in X$, where $\lambda \in\left[0, \frac{1}{\theta(x, y)}\right]$. Then $T$ has a unique fixed point in $X$.

Proof. Let $x_{0} \in X$ be an arbitrary element. Define a sequence $\left\{x_{n}\right\}_{n \geq 1}$ in $X$ as follows.

$$
x_{n+1}=T x_{n}, n \in \mathbb{N} \text {. }
$$

We are to show that $\left\{x_{n}\right\}_{n \geq 1}$ is a Cauchy sequence. For this, we have

$$
\begin{aligned}
\psi\left(x_{n}, x_{n+1}\right)=\psi\left(T x_{n-1}, T x_{n}\right) \preceq & \lambda \psi\left(x_{n-1}, x_{n}\right) \\
& \lambda^{2} \psi\left(x_{n-2}, x_{n-1}\right) \\
& \lambda^{3} \psi\left(x_{n-3}, x_{n-2}\right) \\
& \vdots \\
& \lambda^{n} \psi\left(x_{0}, x_{1}\right) .
\end{aligned}
$$

This implies that

$$
\psi\left(x_{n}, x_{n+1}\right) \preceq \lambda^{n} \psi\left(x_{0}, x_{1}\right) \quad(3.1) .
$$


Now, using the rectangular property of $X$, we get

$$
\begin{aligned}
\psi\left(x_{n}, x_{m}\right) \preceq & \qquad\left(x_{n}, x_{m}\right)\left\{\psi\left(x_{n}, x_{n+1}\right)+\psi\left(x_{n+1}, x_{n+2}\right)+\psi\left(x_{n+2}, x_{m}\right)\right\} \\
& =\theta\left(x_{n}, x_{m}\right) \psi\left(x_{n}, x_{n+1}\right)+\theta\left(x_{n}, x_{m}\right) \psi\left(x_{n+1}, x_{n+2}\right)+\theta\left(x_{n}, x_{m}\right) \psi\left(x_{n+2}, x_{m}\right) \\
& \theta\left(x_{n}, x_{m}\right) \psi\left(x_{n}, x_{n+1}\right)+\theta\left(x_{n}, x_{m}\right) \psi\left(x_{n+1}, x_{n+2}\right) \\
& +\theta\left(x_{n}, x_{m}\right) \theta\left(x_{n+2}, x_{m}\right)\left[\psi\left(x_{n+2}, x_{n+3}\right)+\psi\left(x_{n+3}, x_{n+4}\right)+\psi\left(x_{n+4}, x_{m}\right)\right] \\
& =\theta\left(x_{n}, x_{m}\right) \psi\left(x_{n}, x_{n+1}\right)+\theta\left(x_{n}, x_{m}\right) \psi\left(x_{n+1}, x_{n+2}\right)+\theta\left(x_{n}, x_{m}\right) \theta\left(x_{n+2}, x_{m}\right) \psi\left(x_{n+2}, x_{n+3}\right) \\
& +\theta\left(x_{n}, x_{m}\right) \theta\left(x_{n+2}, x_{m}\right) \psi\left(x_{n+3}, x_{n+4}\right)+\theta\left(x_{n}, x_{m}\right) \theta\left(x_{n+2}, x_{m}\right) \psi\left(x_{n+4}, x_{m}\right) \\
& =\theta\left(x_{n}, x_{m}\right) \psi\left(x_{n}, x_{n+1}\right)+\theta\left(x_{n}, x_{m}\right) \psi\left(x_{n+1}, x_{n+2}\right)+\theta\left(x_{n}, x_{m}\right) \theta\left(x_{n+2}, x_{m}\right) \psi\left(x_{n+2}, x_{n+3}\right) \\
& +\theta\left(x_{n}, x_{m}\right) \theta\left(x_{n+2}, x_{m}\right) \psi\left(x_{n+3}, x_{n+4}\right)+\theta\left(x_{n}, x_{m}\right) \theta\left(x_{n+2}, x_{m}\right) \theta\left(x_{n+4}, x_{m}\right) \psi\left(x_{n+4}, x_{n+5}\right) \\
& \cdot \\
& \cdot \\
& \cdot \\
& \theta\left(x_{n}, x_{m}\right) \psi\left(x_{n}, x_{n+1}\right)+\theta\left(x_{n}, x_{m}\right) \psi\left(x_{n+1}, x_{n+2}\right)+\theta\left(x_{n}, x_{m}\right) \theta\left(x_{n+2}, x_{m}\right) \psi\left(x_{n+2}, x_{n+3}\right) \\
& +\theta\left(x_{n}, x_{m}\right) \theta\left(x_{n+2}, x_{m}\right) \psi\left(x_{n+3}, x_{n+4}\right)+\theta\left(x_{n}, x_{m}\right) \theta\left(x_{n+2}, x_{m}\right) \theta\left(x_{n+4}, x_{m}\right) \psi\left(x_{n+4}, x_{n+5}\right) \\
& +\ldots . .+\theta\left(x_{n}, x_{m}\right) \theta\left(x_{n+2}, x_{m}\right) \theta\left(x_{n+4}, x_{m}\right) \ldots . . \theta\left(x_{m-4}, x_{m}\right) \theta\left(x_{m-2}, x_{m}\right) \psi\left(x_{m-1}, x_{m}\right) .
\end{aligned}
$$

Applying (3.1) to the above inequality, yields

$$
\begin{aligned}
\psi\left(x_{n}, x_{m}\right) & \preceq \theta\left(x_{n}, x_{m}\right) \lambda^{n} \psi\left(x_{0}, x_{1}\right)+\theta\left(x_{n}, x_{m}\right) \lambda^{n+1} \psi\left(x_{0}, x_{1}\right) \\
& +\theta\left(x_{n}, x_{m}\right) \theta\left(x_{n+1}, x_{m}\right) \lambda^{n+2} \psi\left(x_{0}, x_{1}\right)+\theta\left(x_{n}, x_{m}\right) \theta\left(x_{n+1}, x_{m}\right) \lambda^{n+3} \psi\left(x_{0}, x_{1}\right) \\
& +\theta\left(x_{n}, x_{m}\right) \theta\left(x_{n+2}, x_{m}\right) \theta\left(x_{n+4}, x_{m}\right) \lambda^{n+4} \psi\left(x_{0}, x_{1}\right) \\
& +\theta\left(x_{n}, x_{m}\right) \theta\left(x_{n+2}, x_{m}\right) \theta\left(x_{n+4}, x_{m}\right) \lambda^{n+5} \psi\left(x_{0}, x_{1}\right) \\
& \cdot \\
& \cdot \\
& \cdot \\
& +\ldots .+\theta\left(x_{n}, x_{m}\right) \theta\left(x_{n+2}, x_{m}\right) \theta\left(x_{n+4}, x_{m}\right) \ldots . . \theta\left(x_{m-4}, x_{m}\right) \theta\left(x_{m-2}, x_{m}\right) \lambda^{m-2} \psi\left(x_{0}, x_{1}\right) \\
& +\theta\left(x_{n}, x_{m}\right) \theta\left(x_{n+2}, x_{m}\right) \theta\left(x_{n+4}, x_{m}\right) \ldots . . \theta\left(x_{m-4}, x_{m}\right) \theta\left(x_{m-2}, x_{m}\right) \lambda^{m-1} \psi\left(x_{0}, x_{1}\right) . \\
\psi\left(x_{n}, x_{m}\right) & \preceq \theta\left(x_{n}, x_{m}\right)\left[\lambda^{n}+\lambda^{n+1}\right] \psi\left(x_{0}, x_{1}\right) \\
& +\theta\left(x_{n}, x_{m}\right) \theta\left(x_{n+2}, x_{m}\right)\left[\lambda^{n+2}+\lambda^{n+3}\right] d\left(x_{0}, x_{1}\right) \\
& +\theta\left(x_{n}, x_{m}\right) \theta\left(x_{n+2}, x_{m}\right) \theta\left(x_{n+4}, x_{m}\right)\left[\lambda^{n+4}+\lambda^{n+5}\right] \psi\left(x_{0}, x_{1}\right)+ \\
& \cdot \\
& \cdot \\
& \cdot \\
& +\theta\left(x_{n}, x_{m}\right) \theta\left(x_{n+2}, x_{m}\right) \theta\left(x_{n+4}, x_{m}\right) \ldots . . \theta\left(x_{m-4}, x_{m}\right) \theta\left(x_{m-2}, x_{m}\right)\left[\lambda^{m-2}+\lambda^{m-1}\right] \psi\left(x_{0}, x_{1}\right) .
\end{aligned}
$$




$$
\begin{aligned}
\psi\left(x_{n}, x_{m}\right) & \preceq \psi\left(x_{0}, x_{1}\right)\left\{\theta\left(x_{n}, x_{m}\right)\left[\lambda^{n}+\lambda^{n+1}\right]\right. \\
& +\theta\left(x_{n}, x_{m}\right) \theta\left(x_{n+2}, x_{m}\right)\left[\lambda^{n+2}+\lambda^{n+3}\right] \\
& +\theta\left(x_{n}, x_{m}\right) \theta\left(x_{n+2}, x_{m}\right) \theta\left(x_{n+4}, x_{m}\right)\left[\lambda^{n+4}+\lambda^{n+5}\right]+ \\
& \cdot \\
& \cdot \\
& \cdot \\
& \left.+\theta\left(x_{n}, x_{m}\right) \theta\left(x_{n+2}, x_{m}\right) \theta\left(x_{n+4}, x_{m}\right) \ldots . \theta\left(x_{m-4}, x_{m}\right) \theta\left(x_{m-2}, x_{m}\right)\left[\lambda^{m-2}+\lambda^{m-1}\right]\right\} . \\
\psi\left(x_{n}, x_{m}\right) & \preceq \psi\left(x_{0}, x_{1}\right)\left\{\theta\left(x_{1}, x_{m}\right) \theta\left(x_{3}, x_{m}\right) \theta\left(x_{5}, x_{m}\right) \ldots \theta\left(x_{n-1}, x_{m}\right) \theta\left(x_{n}, x_{m}\right)\left[\lambda^{n}+\lambda^{n+1}\right]\right. \\
& +\theta\left(x_{1}, x_{m}\right) \theta\left(x_{3}, x_{m}\right) \theta\left(x_{5}, x_{m}\right) \ldots \theta\left(x_{n}, x_{m}\right) \theta\left(x_{n+2}, x_{m}\right)\left[\lambda^{n+2}+\lambda^{n+3}\right] \\
& +\theta\left(x_{1}, x_{m}\right) \theta\left(x_{3}, x_{m}\right) \ldots \theta\left(x_{n}, x_{m}\right) \theta\left(x_{n+2}, x_{m}\right) \theta\left(x_{n+4}, x_{m}\right)\left[\lambda^{n+4}+\lambda^{n+5}\right]+ \\
& \cdot \\
& \cdot \\
& \cdot \\
& +\theta\left(x_{1}, x_{m}\right) \theta\left(x_{3}, x_{m}\right) \theta\left(x_{5}, x_{m}\right) \ldots \theta\left(x_{n}, x_{m}\right) \theta\left(x_{n+2}, x_{m}\right) \\
& \left.\theta\left(x_{n+4}, x_{m}\right) \ldots . . \theta\left(x_{m-4}, x_{m}\right) \theta\left(x_{m-2}, x_{m}\right)\left[\lambda^{m-2}+\lambda^{m-1}\right]\right\} .
\end{aligned}
$$

By assumption, it is clear that the series $\sum_{n=1}^{\infty}\left[\lambda^{n}+\lambda^{n+1}\right] \prod_{i=1}^{n} \theta\left(x_{i}, x_{m}\right)$ converges by ratio test. For each $m, n \in \mathbb{N}$, take

$$
S=\sum_{n=1}^{\infty}\left[\lambda^{n}+\lambda^{n+1}\right] \prod_{i=1}^{n} \theta\left(x_{i}, x_{m}\right), S_{n}=\sum_{j=1}^{n}\left[\lambda^{j}+\lambda^{j+1}\right] \prod_{i=1}^{j} \theta\left(x_{i}, x_{m}\right) .
$$

Then, fore for all $m>n$, the above inequality implies that

$$
\psi\left(x_{n}, x_{m}\right) \preceq \theta\left(x_{0}, x_{1}\right)\left[S_{m-1}-S_{n}\right] .
$$

By letting $n \rightarrow \infty$ in the above inequality, $\psi\left(x_{n}, x_{m}\right) \preceq 0$, therefore, $\left\{x_{n}\right\}_{n \geq 1}$ is a Cauchy sequence in $X$. Since $X$ is complete, it follows that

$$
x_{n} \rightarrow u \in X \text { as } n \rightarrow \infty .
$$

To show that $u$ is a fixed point of $T$, consider:

$$
\psi(u, T u) \preceq \theta(u, T u)\left[\psi\left(u, x_{n}\right)+\psi\left(x_{n}, x_{n+1}\right)+\psi\left(x_{n+1}, T u\right)\right]
$$


Now, using (3.3), we get

$$
\begin{aligned}
\psi(u, T u) & \preceq \theta(u, T u)[\psi(u, u)+\psi(u, u)+\psi(u, T u)] \\
& =\theta(u, T u)[0+0+\psi(u, T u)] \\
& \preceq \theta(u, T u) \psi(u, T u),
\end{aligned}
$$

from which we have $|1-\theta(u, T u)||\psi(u, T u)| \leq 0$. This implies that $\psi(u, T u)=$ 0 , hence $u$ is a fixed of $T$.

\section{Uniqueness}

Let $v$ be another fixed point of $T$, then

$$
\begin{aligned}
\psi(v, u)=\psi(T v, T u) & \preceq \lambda \psi(u, v) \\
(1-\lambda) \psi(v, u) & \preceq 0 \\
|(1-\lambda) \psi(v, u)| & \leq 0 .
\end{aligned}
$$

This implies that $v=u$ is unique.

Example 3.2. Let $X=[0,1]$. Consider the functions $\theta: X \times X \rightarrow[1, \infty)$ defined by $\theta(x, y)=1+x+y$ and $\psi: X \times X \rightarrow \mathbb{C}$ given by $\psi(x, y)=$ $|x-y|^{2}+i|y-x|^{2}$, for all $x, y \in X$. Then $(X, \psi)$ is a complete complex valued rectangular extended $b$-metric. Now, define $T: X \rightarrow X$ by $T x=$ $\frac{1}{1+x}, x \in X$. To verify (3.1), we note that

$$
\begin{aligned}
& \psi(T x, T y) \preceq \lambda \psi(x, y) \preceq \frac{1}{\theta(x, y)}\left\{|x-y|^{2}+i|y-x|^{2}\right\}, \\
&\left|\frac{1}{1+x}-\frac{1}{1+y}\right|^{2}+\left.i\left|\frac{1}{1+y}-\frac{1}{1+x}\right|^{2} \preceq\right|^{2} \preceq \frac{1}{\theta(x, y)}\left\{|x-y|^{2}+i|y-x|^{2}\right\}, \\
&\left|\frac{y-x}{(1+x)(1+y)}\right|^{2}+i\left|\frac{x-y}{(1+x)(1+y)}\right|^{2} \\
& \frac{1}{(1+x)^{2}(1+y)^{2}}\left\{|x-y|^{2}+i|y-x|^{2}\right\} \preceq \frac{1}{1+x+y}\left\{|x-y|^{2}+i|y-x|^{2}\right\}, \\
& 1+x+y \leq(1+x+y+x y)^{2}
\end{aligned}
$$

hold for all $x, y \in X$, Thus, all the conditions of Theorem (3.1) are satisfied, and hence $T$ has a unique fixed point in $X$.

In the following, we establish a Kannan-type fixed point result for complexvalued rectangular extended $b$-metric space.

Theorem 3.3. Let $(X, \psi)$ be a complete complex valued rectangular extended b-metric space with $\theta: X \times X \rightarrow[1, \infty]$, and $T: X \rightarrow X$ be a mapping satisfying:

$$
\psi(T x, T y) \preceq \lambda[\psi(x, T x)+\psi(y, T y)]
$$

for all $x, y \in X$, where $\lambda \in\left[0, \frac{1}{\theta(x, y)}\right]$. Then $T$ has a unique fixed point in $X$. 
Proof. Let $x_{0} \in X$ be an arbitrary point. we define a sequence $\left\{x_{n}\right\}_{n \geq 1}$ by $x_{n+1}=T x_{n}$ for $n \in \mathbb{N}$. Now, using (3.4)

$$
\begin{aligned}
\psi\left(x_{n}, x_{n+1}\right) & \preceq \psi\left(T x_{n-1}, T x_{n}\right) \\
& \preceq \lambda\left[\psi\left(x_{n-1}, T x_{n-1}\right)+\psi\left(x_{n}, T x_{n}\right)\right] \\
& \preceq \lambda\left[\psi\left(x_{n-1}, x_{n}\right)+\psi\left(x_{n}, x_{n+1}\right)\right] \\
(1-\lambda) \psi\left(x_{n}, x_{n+1}\right) & \preceq \lambda \psi\left(x_{n-1}, x_{n}\right) \\
\psi\left(x_{n}, x_{n+1}\right) & \preceq\left\{\frac{\lambda}{1-\lambda}\right\} \psi\left(x_{n-1}, x_{n}\right) \\
& \preceq\left\{\frac{\lambda}{1-\lambda}\right\}^{2} \psi\left(x_{n-2}, x_{n-1}\right) \\
& \cdot \\
& \cdot \\
& \preceq\left\{\frac{\lambda}{1-\lambda}\right\}^{n} \psi\left(x_{0}, x_{1}\right) .
\end{aligned}
$$

Let $h=\frac{\lambda}{1-\lambda}<1$. Then,

$$
\begin{aligned}
\psi\left(x_{n}, x_{m}\right)=\psi\left(T x_{n-1}, T x_{m-1}\right) & \preceq \lambda\left[\psi\left(x_{n-1}, T x_{n-1}\right)+\psi\left(x_{m-1}, T x_{m-1}\right)\right] \\
& \preceq \lambda\left[\psi\left(x_{n-1}, x_{n}\right)+\psi\left(x_{m-1}, x_{m}\right)\right] \\
& \preceq \lambda\left[h^{n-1} \psi\left(x_{0}, x_{1}\right)+h^{m-1} \psi\left(x_{0}, x_{1}\right)\right] .
\end{aligned}
$$

By letting $m, n \rightarrow \infty$, we get $h^{n-1} \rightarrow 0$ and $h^{m-1} \rightarrow 0$, and so,

$$
\psi\left(x_{n}, x_{m}\right) \preceq \lambda\left[h^{n-1} \psi\left(x_{0}, x_{1}\right)+h^{m-1} \psi\left(x_{0}, x_{1}\right)\right] \rightarrow 0
$$

This implies that $\psi\left(x_{n}, x_{m}\right) \rightarrow 0$, hence $\left\{x_{n}\right\}_{n \geq 1}$ is a Cauchy sequence. The completeness of $X$ implies that there exist $u \in X$ such that $x_{n} \rightarrow u(n \rightarrow \infty)$. 
To see that $u \in X$ is a fixed point of $T$, consider:

$$
\begin{aligned}
\psi(u, T u) & \preceq \theta(u, T u)\left[\psi\left(u, x_{n}\right)+\psi\left(x_{n}, x_{n+1}\right)+\psi\left(x_{n+1}, T u\right)\right] \\
& \preceq \theta(u, T u)\left[\psi\left(u, T x_{n-1}\right)+\psi\left(x_{n}, x_{n+1}\right)+\psi\left(T x_{n}, T u\right)\right] \\
& \preceq \theta(u, T u) \psi\left(u, T x_{n-1}\right)+\theta(u, T u) \psi\left(x_{n}, x_{n+1}\right)+ \\
& \theta(u, T u) \lambda\left[\psi\left(x_{n}, T x_{n}\right)+\psi(u, T u)\right] \\
& \preceq \theta(u, T u) \psi\left(u, T x_{n-1}\right)+\theta(u, T u) h^{n} \psi\left(x_{0}, x_{1}\right)+\lambda \theta(u, T u) \psi\left(x_{n}, x_{n-1}\right)+ \\
& \theta(u, T u) \lambda \psi(u, T u) \\
& \preceq \theta(u, T u) \psi\left(u, T x_{n-1}\right)+\theta(u, T u) h^{n} \psi\left(x_{0}, x_{1}\right)+\lambda \theta(u, T u) h^{n} \psi\left(x_{0}, x_{1}\right)+ \\
& \lambda \theta(u, T u) \psi(u, T u) \\
(1-\lambda \theta(u, T u)) \psi(u, T u) & \preceq \theta(u, T u) \psi\left(u, T x_{n-1}\right)+\theta(u, T u) h^{n} \psi\left(x_{0}, x_{1}\right)+\lambda \theta(u, T u) h^{n} \psi\left(x_{0}, x_{1}\right) \\
\psi(u, T u) & \preceq \frac{\theta(u, T u)}{1-\lambda \theta(u, T u)} \psi\left(u, T x_{n-1}\right)+\frac{\theta(u, T u)}{1-\lambda \theta(u, T u)} h^{n} \psi\left(x_{0}, x_{1}\right)+ \\
& \frac{\lambda \theta(u, T u)}{1-\lambda \theta(u, T u)} h^{n} \psi\left(x_{0}, x_{1}\right) .
\end{aligned}
$$

By letting $n \rightarrow \infty$ in the above expression, we get

$$
\left(1-\frac{\theta(T u, u)}{1-\lambda \theta(T u, u)}\right) \psi(u, T u) \preceq 0
$$

This implies that

$$
\begin{gathered}
\psi(u, T u) \preceq 0 \\
|\psi(u, T u)| \leq 0 \\
\psi(u, T u)=0 \\
u=T u .
\end{gathered}
$$

\section{Uniqueness}

Let $u^{*}$ be another fixed point of $T$. Then,

$$
\begin{aligned}
\psi\left(u, u^{*}\right)=\psi\left(T u, T u^{*}\right) & \preceq \lambda\left[\psi(u, T u)+\psi\left(u^{*}, T u^{*}\right)\right] \\
& \preceq \lambda\left[\psi(u, u)+\psi\left(u^{*}, u^{*}\right)\right] \\
\psi\left(u, u^{*}\right) & \preceq 0 \\
\left|\psi\left(u, u^{*}\right)\right| & \leq 0 \\
\psi\left(u, u^{*}\right) & \leq 0,
\end{aligned}
$$

from which it follows that $u=u^{*}$.

Example 3.4. $X=\{1,2,3,4\}$. Define $\psi: X \times X \rightarrow \mathbb{C}$ as follows:

$\psi(1,2)=\psi(2,1)=3 i$

$\psi(2,3)=\psi(3,2)=\psi(1,3)=\psi(3,1)=1 i$ 


$$
\begin{gathered}
\psi(1,4)=\psi(4,1)=\psi(2,4)=\psi(4,2)=\psi(3,4)=\psi(4,3)=4 i . \\
\theta: X \times X \rightarrow[1, \infty) \text { by } \\
\theta(x, y)=1+x+y .
\end{gathered}
$$

Thus $(X, \psi)$ is a complex valued rectangular extended $b$-metric space. Now, define a mapping $T: X \rightarrow X$ as

$$
T x= \begin{cases}3, & \text { if } x \neq 4 \\ 1, & \text { if } x=4 .\end{cases}
$$

By using the definition, we get $\psi(T 1, T 2)=\psi(T 1, T 3)=\psi(T 2, T 3)=0 i$ and in the remaining points of $X$, we have $\psi(T x, T y)=1 i,[\psi(x, T x)+$ $\psi(y, T y)] \geq 4 i$. Hence for, $\lambda=\frac{1}{2}$ all the assertion of theorem (3.4) are satisfied and thus 3 is the unique fixed point $T$.

Remark 3.5. Clearly, the function $\psi$ in Examples 3.2 and 3.4 are not rectangular $b$-metrics. Hence, the result of George et al. [22, Theorem 2.1] is not applicable to these examples. It is also obvious that Theorem 3.1 is a generalization of Theorem 2.12. Similarly, all the results of Ullah et al. [23], being presented in the setting of multivalued mappings are not comparable with all the results established herein.

\section{Applications}

In this section, we give an existence theorem for Fredholm integral equation as a usability of Theorem 3.1. Let $X=C([a, b], \mathbb{C})$ be the space of all

continuous complex-valued functions defined on closed interval $[a, b]$. Define

$$
\begin{aligned}
\psi & : X \times X \rightarrow \mathbb{C} b y \\
\psi(x, y) & =\sup _{t \in[a, b]}|x(t)-y(t)| e^{i t},
\end{aligned}
$$

and the function $\theta: X \times X \rightarrow[1, \infty)$ by

$$
\theta(x, y)=|x(t)|+|y(t)|+2 .
$$

Then $X=C([a, b], \mathbb{C})$ is complete complex valued rectangular extended $b$-metric space. Consider the Fredholm integral equation:

$$
x(t)=\int_{a}^{b} M(t, s, x(s)) d s+g(t), \quad t, s \in[a, b]
$$

(4.2) where $g:[a, b] \rightarrow \mathbb{R}$ and $M:[a, b] \times[a, b] \times \mathbb{R} \rightarrow \mathbb{R}$ are continuous.

We investigate the existence of solution of problem (4.1) under the following assumption: 
Theorem 4.1. Problem (4.1) has a unique solution in $X=C([a, b], \mathbb{C})$ if there exists a number $\lambda \in\left[0, \frac{1}{\theta(x, y)}\right]$ such that for all $x, y \in X$,

$$
|M(t, s, x)-M(t, s, y)| \leq \Omega|x-y|,
$$

for each $(s, t) \in[a, b] \times[a, b]$, where $\Omega=\frac{\lambda}{b-a}$.

Proof. First, we convert (4.1) into a fixed point problem. For this, define

$$
\begin{aligned}
T: X \rightarrow X \text { by } & \\
T x(t) & =\int_{a}^{b} M(t, s, x(s)) d s+g(t), \quad t, s \in[a, b] .
\end{aligned}
$$

Clearly, the fixed point of the mapping defined by (4.3) is a solution of problem (4.1). From Condition (3.1), we have

$$
\begin{aligned}
\psi(T x(t), T y(t))= & \sup _{t \in[a, b]}|T x(t)-T y(t)| e^{i t} \\
= & \sup _{t \in[a, b]}\left|\int_{a}^{b} M(t, s, x(s)) d s+g(t)-\int_{a}^{b} M(t, s, y(s)) d s-g(t)\right| e^{i t} \\
\preceq & \int_{a}^{b} \sup _{t \in[a, b]}|M(t, s, x(s))-M(t, s, y(s))| e^{i t} d s \\
& =\sup _{t \in[a, b]}|M(t, s, x(s))-M(t, s, y(s))| e^{i t} \int_{a}^{b} d s \\
& \preceq\left(\frac{\lambda}{b-a}\right)(b-a) \psi(x(t), y(t)) \\
& =\lambda \psi(x(t), y(t)) .
\end{aligned}
$$

Thus, all the conditions of Theorem 3.1 are satisfied. Consequently, Problem (4.1) has a unique solution in $X$.

\section{Competing Interests}

The authors declare that they have no competing interests.

\section{Acknowledgments}

The authors are thankful to the editors and the anonymous reviewers for their valuable suggestions and comments that helped to improve this manuscript.

\section{REFERENCES}

[1] J. Ahmad, C. Klin-Eam, and A. Azam, Common fixed points for multivalued mappings in complex valued metric spaces with applications. In Abstract and Applied Analysis, 2013. 
[2] J. Ahmad, N. Hussain, A. Azam and M. Arshad, Common fixed point results in complex valued metric with application to system of integral equations. Journal of Nonlinear and Convex Analysis, 29(5):855-871, 2015.

[3] M. Aiman, Some common fixed point theorems in complex valued b-metric spaces, The Scientific World Journal, 2014.

[4] A. Azam, B. Fisher and M. Khan, Common fixed point theorems in complex valued metric spaces Numerical Functional Analysis and Optimization, 32(3):243-25, 2011.

[5] A. Azam, J. Ahmad and P. Kumam, Common fixed point theorems for multi-valued mappings in complex-valued metric spaces, Numer. Funct. Anal. Optim, 33(5):590600, 2012.

[6] A. Azam, J. Ahmad, and P. Kumam, Common fixed point theorems for multi-valued mappings in complex-valued metric spaces, Journal of Inequalities and Applications, 1, 2013.

[7] A. Azam,and N. Mehmood, Multivalued fixed point theorems in tvs-cone metric spaces, Fixed Point Theory and Applications, 1, 184, 2013.

[8] I. A. Bakhtin, The contraction principle in quasimetric spaces, Functional Analysis, 30, 26-37, 1989.

[9] S. Banach, Sur les operation dans les ensembles abstraits et applications aux equations integrales, Fund. Math, (French), 133-181, 1922.

[10] N. Bourbaki, Topologie Generale; Herman: Paris, France, 1974.

[11] S. Czerwik, Contraction mappings in b-metric spaces, Acta Mathematica et Informatica Universitatis Ostraviensis, 1(1), 5-11, 1993.

[12] A. K. Dubey, R. Shukla and R. P. Dubey, Some fixed point theorems in complex valued b-metric spaces. Journal of Complex Systems, 2015.

[13] A. K. Dubey, Common fixed point results for contractive mappings in complex valued b-metric spaces, Nonlinear Functional Analysis and Applications, vol. 20, 257-268, 2015.

[14] A. K. Dubey, Complex Valued-Metric Spaces and Common Fixed Point Theorems under Rational Contractions. Journal of Complex analysis, 2016.

[15] T. Kamran, M. Samreen, Q., UL Ain, A generalization of $b$-metric space and some fixed point theorems, Mathematics, 5(2), 19, 2017.

[16] R. Kannan, Some results on fixed points, Bull Calcutta Math Soc, 60:71--76, 1968.

[17] E. Karapinar, A Short Survey on the Recent Fixed Point Results on $b$-Metric Spaces, Constructive mathematical Analysis, 1(1), 2018.

[18] J. Kumar, Common Fixed Point Theorem for Generalized Contractive Type Maps on Complex Valued b-Metric Spaces, International Journal of Mathematical Analysis, 9, 2327-2334, 2015.

[19] M. A. Kutbi, E. Karapınar, J. Ahmad, and A.Azam, Some fixed point results for multi-valued mappings in b-metric spaces, Journal of Inequalities and Applications, 1: 126,2014

[20] P. Rao, R. Swamy, and J. R. Prasad, A common fixed point theorem in complex valued b-metric spaces, Bulletin of Mathematics and Statistics Research, 1: 1-8, 2013.

[21] M. R. Taskovic, A generalization of Banach's contraction principle, Publ. Inst. Math, 37:179-191, 1978.

[22] R. George, S. Radenovic, P.K. Reshma, S. Shukla, Rectangular b-metric space and contraction principles, J. Nonlinear Sci. Appl, 8(6):1005-1013, 2015.

[23] N. Ullah, M.S. Shagari, and A. Azam, Fixed Point Theorems in Complex Valued Extended b-Metric Spaces, Moroccan Journal of Pure and Applied Analysis, 5(2): 140-163, 2019

[24] Z.D. Mitrovic, S. Radenovic, On Meir-Keeler contraction in Branciari b-metric spaces, 9(3):34-41, 2018. 
(Naimat Ullah) Department of Mathematics, Visiting Faculty, University of MiAnWALi, PAKISTAN

Email address: naimat0347@gmail.com

(Mohammed Shehu Shagari) Department of Mathematics, Faculty of Physical Sciences, Ahmadu Bello University, Nigeria

Email address: shagaris@ymail.com 